\title{
Relationship between HOX gene and pediatric congenital clubfoot
}

\author{
YUANHUI WANG \\ Department of the Bone and Joint Surgery, The Affiliated Hospital of \\ Southwest Medical University, Luzhou, Sichuan 646000, P.R. China
}

Received August 3, 2017; Accepted January 19, 2018

DOI: $10.3892 /$ etm.2018.6013

\begin{abstract}
The relationship between transcription factor homeobox gene (HOX gene) and pediatric congenital clubfoot (CCF) was studied. The CCF group comprised 35 cases of children, and the control group compised 34 cases of children without congenital malformation. The levels of inducible nitric oxide synthase (iNOS) and nitric oxide (NO) in the serum of the control and CCF groups were measured using iNOS and NO kits. Interleukin-1 $\beta$ (IL-1 $\beta$ ), IL-6 and tumor necrosis factor- $\alpha$ (TNF- $\alpha$ ) related to inflammation in the tissues of both groups were detected by reverse transcription-polymerase chain reaction (RT-PCR). Fatty acid synthase (Fas), Fas ligand (FasL) and Bcl-2-associated X (Bax) related to apoptosis as well as the expression of HOX mRNA, the expression of HOX in the control and CCF groups was detected by western blot analysis, and the differential expression of HOX in the control and CCF groups was statistically analyzed. Results of the kit detection showed that the expression of iNOS and NO in the CCF group were significantly higher than those in the control group, indicating that severe oxidative damage occurred in the CCF group. The results of detecting inflammatory factors and apoptosis by RT-PCR showed that the expression of IL-1 $\beta$, IL- 6 , TNF- $\alpha$, Fas, FasL and Bax mRNA in the CCF group was significantly higher than that in the control group, indicating pathogenesis of CCF was related to inflammation and apoptosis. RT-PCR and western blot analysis revealed HOX was highly expressed in the tissues of CCF, and the expression quantity was significantly stronger than that in the control group. The result of analysis of variance showed that the expression differences of HOX in normal and CCF tissues were statistically significant $(\mathrm{P}<0.01)$. Abnormal expression of HOX was closely related to the occurrence and development of $\mathrm{CCF}$, indicating that
\end{abstract}

Correspondence to: Dr Yuanhui Wang, Department of the Bone and Joint Surgery, The Affiliated Hospital of Southwest Medical University, 25 Taiping Street, Jiangyang, Luzhou, Sichuan 646000 , P.R. China

E-mail: yuanhui8526@163.com

Key words: homeobox gene, pediatric, congenital clubfoot
HOX has important research value in $\mathrm{CCF}$ and this functional mechanism is related to oxidative damage, inflammation and apoptosis. Expression of HOX therefore shows promise as an indicator of CCF diagnosis and treatment.

\section{Introduction}

Congenital clubfoot (CCF) is a common congenital malformation that seriously endangers children's health (1). The pathogenesis of CCF is unclear. Previous findings showed that common impacts of multiple factors lead to the occurrence of CCF $(2,3)$. At present, an increasing number of studies have focused on CCF. With the development of genetics and genetic technology, it has been found that multiple genes play an important role in the occurrence and development of CCF (4).

Transcription factor homeobox gene ( $\mathrm{HOX}$ gene) belongs to the homeobox family, which constitutes genes that specifically regulate and control biological form and structure in living organism $(5,6)$. Previous findings have shown that HOX gene mutation is closely related to the occurrence and development of $\mathrm{CCF}(7)$.

The present study explored the pathogenetic process of CCF by detecting the levels of iNOS and NO, as well as the factors associated with inflammation and apoptosis, and the expression of HOX gene in CCF was detected by RT-PCR and western blot analysis, in order to provide guidance for genetic diagnosis, precaution and treatment of CCF.

\section{Patients and methods}

Patient data. Patients included in the present study comprised children who were hospitalized and had undergone operations in Jiangyang Hospital between April 2009 and May 2015. All the child patients had clinical symptoms of typical CCF. If combined with other malformations the patients were excluded by examination and operational definite diagnosis. The CCF group comprised 35 cases of children with CCF (male, 18 cases; female, 17 cases), aged 0.6-9 years, with an average age of 3.4 years. Muscle specimens were from the children who had undergone CCF operations, and $100 \mathrm{mg}$ lower leg gastrocnemius was collected for the study. The control group comprised 34 cases of children without congenital malformation who had trauma operations of lower legs or had taken out internal fixation after the traumas of lower legs 
(male, 20 cases; female, 14 cases). These childrend were aged 1.1-10 years, with an average age of 6.8 years. Approximately $100 \mathrm{mg}$ lower leg gastrocnemius was collected as muscle specimens from the same location as the CCF group.

Signed informed consent forms were obtained from the children's family for the collection and use of the specimens. The study was approved by the Ethics Committee of the Affiliated Hospital of Southwest Medical University (Luzhou, China).

Primary reagents. iNOS and NO detection kit (Jiancheng, Nanjing, China); bicinchoninic acid (BCA) protein assay kit (Biyuntian, Shanghai, China); TRIzol total RNA extraction kit; reverse transcription-polymerase chain reaction (RT-PCR) reverse transcription kit (both from Tiangen, Beijing, China); glyceraldehyde-3-phosphate dehydrogenase (GAPDH) and HOX monoclonal antibodies and secondary antibodies (CST, Boston, MA, USA) were used in the study.

\section{Experimental methods}

Measurement of iNOS. The serum samples of the patients in the control and CCF groups were collected. The levels of iNOS and NO in the sera were measured and the results of the data were analyzed in accordance with the protocols provided by Nanjing Jiancheng Biology Co., Ltd.

RT-PCR analysis. RT-PCR analysis was carried out using the following steps:

a) Extraction of total RNA. The collected tissues were rapidly transferred to Eppendorf (EP) tubes that contained $1.5 \mathrm{ml}$ RNAiso Plus extracting solution (the tissues needed homogenizing thoroughly). After $5 \mathrm{~min}$ standing, the samples were disintegrated completely and centrifuged for $5 \mathrm{~min}$ at $12,000 \mathrm{x} g$ at $4^{\circ} \mathrm{C}$. The supernatan $\mathrm{t}$ was carefully absorbed to be transferred to new EP tubes. Chloroform $(0.2 \mathrm{ml})$ was added to the supernatant, and then mixed evenly. After $5 \mathrm{~min}$ standing at room temperature, the samples were centrifuged for $15 \mathrm{~min}$ at $12,000 \mathrm{x} \mathrm{g}$ at $4^{\circ} \mathrm{C}$, the supernatant was absorbed, and then the same volume of isopropanol was added, mixed evenly, and left to stand for $10 \mathrm{~min}$ at room temperature. The samples were then centrifuged for $10 \mathrm{~min}$ at $12,000 \mathrm{x}$ g at $4^{\circ} \mathrm{C}$, the supernatant was discarded carefully, and the sediment was preserved. Subsequently, $1 \mathrm{ml}$ 75\% ethyl alcohol was added, and the samples were mixed evenly, followed by centrifugation for $5 \mathrm{~min}$ at $12,000 \mathrm{x}$ g at $4^{\circ} \mathrm{C}$. The supernatant was carefully discarded, and the process was repeated. After the RNA sediment was washed, the liquid was discarded. Finally, when the ethyl alcohol volatilized completely, the RNase-free water was added.

b) Purity analysis and content calculation of total RNA. Total RNA solution ( $4 \mu \mathrm{l}$ ) was diluted with $1 \mathrm{ml}$ RNase-free water, and the absorbance values were measured at wavelengths of 260, 280 and $320 \mathrm{~nm}$. If optical density $\left(\mathrm{OD}_{260} / \mathrm{OD}_{280}\right)$ was between 1.8 and 2.2, it showed that the purity of sample RNA met the requirement. RNA concentration $(\mu \mathrm{g} / \mu \mathrm{l})=\left(\mathrm{OD}_{260}-\mathrm{OD}_{320}\right) \times$ dilution ratio $\mathrm{x} 0.04$.

c) Reverse transcription. After partial RNA solution was diluted with RNase-free water to get $1 \mu \mathrm{g} / \mu \mathrm{l}$ solution, reverse transcription solution was prepared in accordance with the instructions of the PrimeScript ${ }^{\circledR}$ RT reagent kit with gDNA eraser kit, into which the corresponding RNA samples were
Table I. Primer sequences of related genes were analyzed by RT-PCR analysis.

\begin{tabular}{llc}
\hline Gene names & \multicolumn{2}{c}{ Primer sequences } \\
\hline IL-1 $\beta$ & 5'-3' & CTGAGCACCTTCTTTCCCTTCA \\
& 3'-5' & TGGACCAGACATCACCAAGCT \\
IL-6 & 5'-3' & TGGCTGAAAAAGATGGATGCT \\
& 3'-5' & TCTGCACAGCTCGGCTTGT \\
TNF- $\alpha$ & 5'-3' & TGTAGCCCATGTTGTAGCAAACC \\
& 3'-5' & GAGGACCTGGGAGTAGATGAGGTA \\
Fas & 5'-3' & ACATGGACAAGAACCATTATGCTGA \\
& 3'-5' & CTGGTTTGCACTTGCACTTGGTA \\
FasL & 5'-3' & CATGCAGCAGCCCATGAATTAC \\
& 3'-5' & CTCTAGGCCCACAAGATGGACAG \\
Bax & 5'-3' & CAGGATGCGTCCACCAAGAA \\
& 3'-5' & CGTGTCCACGTCAGCAATCA \\
HoX & $5 '-3 '$ & GTGATCTGTAATCCCTATGAG \\
& 3'-5' & TFGTTACCCTGCATTACGATG \\
& 5'-3' & GAGCCGGGAAATCGTGCGT \\
& 3'-5' & GGAAGGAAGGCTGGAAGATG
\end{tabular}

PCR, polymerase chain reaction; IL-1 $\beta$, interleukin- $1 \beta$; TNF- $\alpha$, tumor necrosis factor- $\alpha$; Fas, fatty acid synthase; FasL, Fas ligand; Bax, Bcl-2-associated X; HOX, homeobox.

added, and reverse transcription was performed to obtain cDNA. The obtained cDNA was kept at $-20^{\circ} \mathrm{C}$.

d) $q P C R$ analysis. According to the protocol of the SYBR $^{\circledR}$ Premix Ex Taq ${ }^{\mathrm{TM}}$ II (Tli RNaseH Plus) kit, the levels of mRNA were measured. Primer sequences of corresponding RNA are shown in Table I.

Western blot analysis. The collected tissues of the control and CCF groups were washed respectively with ice-cold physiological saline. The protein concentrations were measured by BCA protein assay kit, and preserved for standby application after being packed separately at $-80^{\circ} \mathrm{C}$. The procedure was performed according to the protocol of the total protein extration kit. Immunoprecipitation (IP) lysate containing phenylmethanesulfonyl fluoride (PMSF) and protease inhibitor was added, and the tissues were placed on ice for full grinding. After tissue homogenate was centrifuged for $10 \mathrm{~min}$ at $4^{\circ} \mathrm{C}$ and $12,000 \mathrm{x} \mathrm{g}$, the supernatant was taken; after it was centrifuged for $20 \mathrm{~min}$ again at $4^{\circ} \mathrm{C}$ and $12,000 \mathrm{x} \mathrm{g}$, the supernatant was taken. Then, after the supernatant and protein qualification were carried out according to the protocol of the protein kit, the protein samples containing an equal quantity of total protein were loaded and placed onto sample loading wells, and electrophoresed at constant voltage of $220 \mathrm{~V}$, and the electrophoresis stopped when the bromophenol blue reached the bottom of the gel. According to molecular weight of target protein, the gel was cut and placed into transfer buffer. Polyvinylidene fluoride (PVDF) membrane was cut to keep the target band only, and the PVDF membrane was soaked in methyl alcohol for $10 \mathrm{sec}$. The PVDF membrane and filter paper were placed in a transfer buffer and then a transmembrane device in 

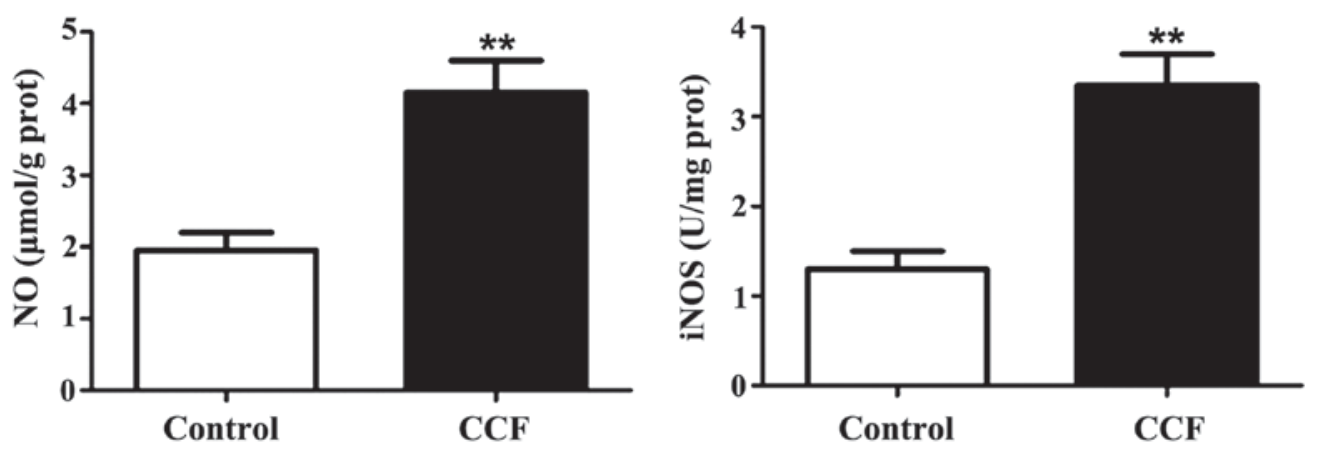

Figure 1. Expression of inducible nitric oxide synthase (iNOS) and nitric oxide (NO) in control group and congenital clubfoot (CCF) group. Comparison with control group, ${ }^{* *} \mathrm{P}<0.05(\mathrm{n}=3)$.

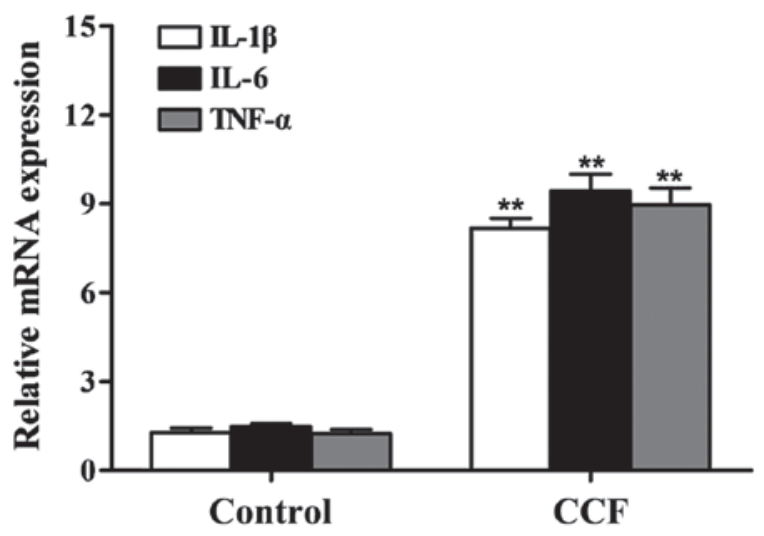

Figure 2. Expression of interleukin-1 $\beta$ (IL-1 $\beta$ ), IL-6 and tumor necrosis factor- $\alpha$ (TNF- $\alpha)$ in the control group and congenital clubfoot (CCF) group. Comparison with control group, ${ }^{* *} \mathrm{P}<0.05(\mathrm{n}=3)$.

the order of positive electrode, filter paper, PVDF membrane, gel, filter paper, negative electrode, and PVDF membrane was transferred for appropriate time at constant voltage of $110 \mathrm{~V}$, for the protein of the gel to transfer to the PVDF membrane. The PVDF membrane to which protein was adhered was placed in 5\% skim milk powder, and then agitated and sealed for $3 \mathrm{~h}$ at room temperature. The membrane was then placed in rabbit anti-human GAPDH monoclonal antibody (dilution, 1:1,000; cat. no. 2118) and rabbit anti-human HOX primary monoclonal antibodies (dilution, 1:1,000; cat. no. 90944) for incubation overnight at $4^{\circ} \mathrm{C}$. The next day, the PVDF membrane was completely washed with Tween-20/Tris-buffered salt (TTBS) (10 $\mathrm{min} /$ time, 3 times), and then goat anti-rabbit secondary polyclonal antibody (dilution, 1:2,000; cat. no 7074) were added and incubated for $1 \mathrm{~h}$ at room temperature. After washing with TTBS (10 min/time, 3 times), enhanced chemiluminescence (ECL) color-substrate solution was added dropwise for color development photography.

Statistical analysis. Experimental data were expressed with mean \pm standard deviation, and statistical analysis for experimental results was made by Statistical Product and Service Solutions 17.0 (SPSS 17.0; Chicago, IL, USA). Mean comparison between the two groups were obtained using the Student's t-test. For mean comparisons among the groups one-way analysis of variance (ANOVA) was used. SNK test was used as post hoc test. Statistical significance was set at $\mathrm{P}<0.05$.

\section{Results}

Measured results of iNOS and NO. As shown in Fig. 1, the levels of iNOS and NO between the control and CCF groups were significantly different. The levels of iNOS and NO in the CCF group were significantly higher than those in the control group, which indicated that obvious oxidative damage occurred in the CCF group.

$R T-P C R$ results of the factors associated with inflammation. Total RNA was extracted respectively from tissue samples of the control and CCF groups. After RT-PCR, the expression of IL-1 $\beta$, IL- 6 and TNF- $\alpha$ in CCF group was significantly higher than those in control group (Fig. 2). It could thus be seen that excessive expression of inflammatory factors generated serious inflammatory reaction in pathogenic process of $\mathrm{CCF}$.

RT-PCR results of the factors associated with apoptosis. As shown in Fig. 3, the expression of Fas, FasL and Bax in CCF group was significantly higher than that in the control group. Thus, the excessive expression of the factors related to apoptosis resulted in serious tissue apoptosis and damage in pathogenic process of $\mathrm{CCF}$.

$R T-P C R$ result of HOX $m R N A$. The expression of HOX RNA in tissue samples of the control and CCF groups was detected, and the results are shown in Fig. 4. As shown the expression quantity of HOX in CCF group was significantly higher than that in the control group, which indicated that the expression of HOX mRNA in CCF was very high and was associated with the development of CCF.

Expression of HOX protein in the control and CCF groups. Results of the western blot analysis revealed the expression of HOX protein in the tissues of the control and CCF groups. As shown in Fig. 5, HOX protein was expressed highly in the tissues of CCF group, and the expression quantity was greater than that in control group.

\section{Discussion}

$\mathrm{CCF}$ is a common congenital malformation that threatens children's health and seriously impacts on foot function (8). At present, the pathogenesis of CCF is not very clear, there are main theories such as germplasm defect theory, developmental 

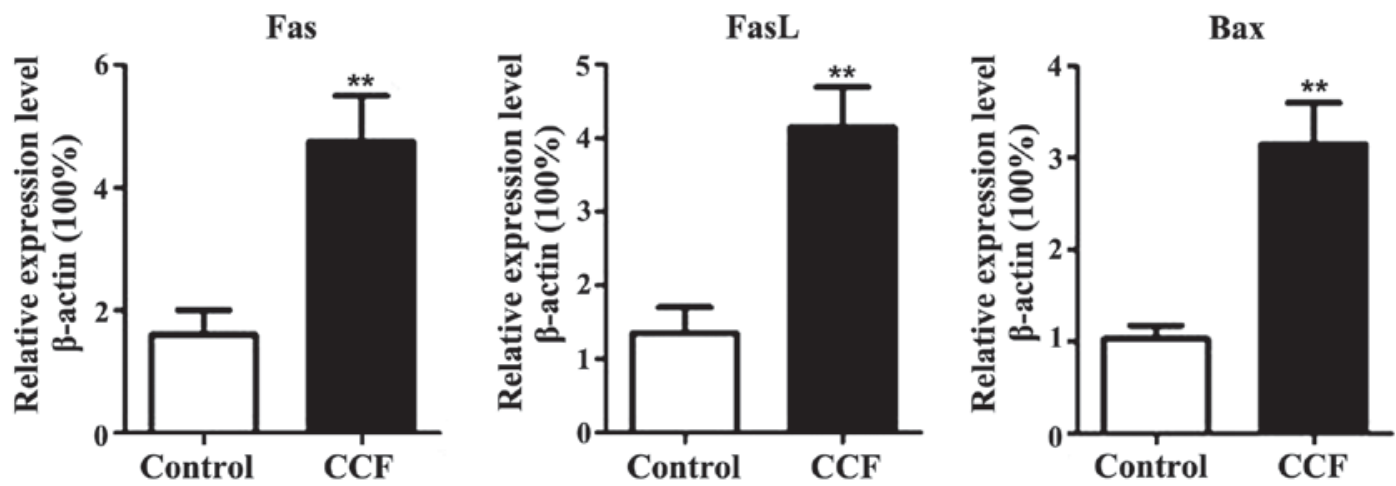

Figure 3. Expression of Fas, FasL and Bax in control group and congenital clubfoot (CCF) group. Comparison with control group, ${ }^{* * *} \mathrm{P}<0.05$ ( $\mathrm{n}=3$ ).

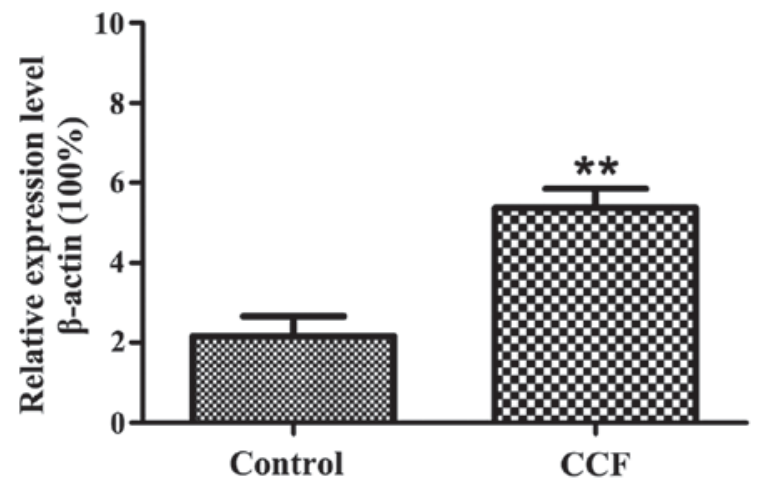

Figure 4. Expression of homeobox (HOX) mRNA in the control group and congenital clubfoot (CCF) group. Comparison with control group, ${ }^{* *} \mathrm{P}<0.05(\mathrm{n}=3)$.

block theory, theories of neurogenic myogenic and angiogenic abnormality in embryonic period and theory of fiber curliness denaturation (9-11). Currently, the most respected theory is the theory of neuromuscular lesion (12). In recent years, with the rapid development of epidemiologic studies, we identified that genetic factors play an important role (13) in the pathogenesis process of CCF.

The development process of limbs is a very complex process regulated and controlled by multiple genes (14). The development process refers to the sequence of successive expression of multiple genes within different periods and different spaces (15). Transcription factor $H O X$ gene is an effective gene in the development of limbs that has been extensively studied (16). HOX gene plays a very important role in many parts of the body, including nerves, muscles, bones and blood vessels. Thus, diseases on limbs can be caused once HOX gene has mutations or abnormal expression (17). The occurrence of CCF may be the abnormal expression of $\mathrm{HOX}$ gene in the development process of limbs, and then abnormal embryonic development is caused to lead to CCF (18). Some studies showed that $H O X$ gene adjusted the factors associated with apoptosis, thus it indicates that $H O X$ gene may be the important susceptibility gene of CCF $(19,20)$.

This study included 35 cases of child patients with CCF as the $\mathrm{CCF}$ group, and 34 cases of children with congenital malformation as the control group. iNOS and NO kits were used to measure the levels of iNOS in the sera of the control and CCF groups. IL-1 $\beta$, IL-6, TNF- $\alpha$, Fas, FasL, Bax and the expression of HOX mRNA in the tissues of both groups were detected by RT-PCR. The expression of HOX in the control and CCF groups was detected via western blot analysis. Experimental results showed that oxidative damage, inflammatory factors and apoptotic factors appeared in CCF group. RT-PCR and western blot analysis showed that, HOX was highly expressed in CCF group, where the expression quantity of HOX was significantly higher than that in normal tissues.

In conclusion, the present study made some progress in determining the aetiological agent of CCF and genetic expression of HOX. However, more follow-up studies should be
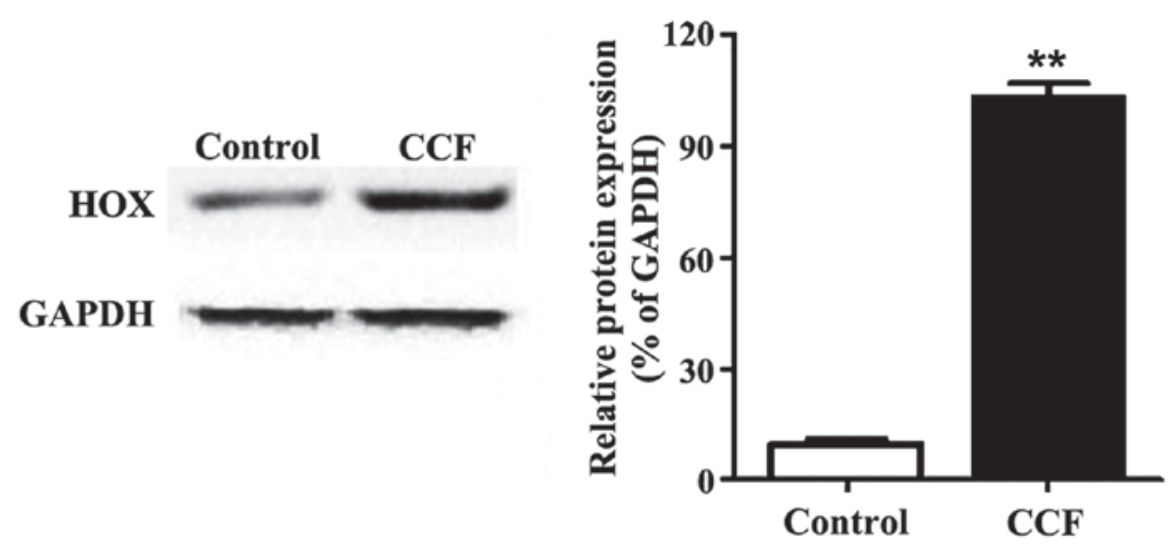

Figure 5. Expression of homeobox $(\mathrm{HOX})$ protein in control group and congenital clubfoot $(\mathrm{CCF})$ group. Comparison with control group, ${ }^{* *} \mathrm{P}<0.05(\mathrm{n}=3)$. 
conducted because the pathogenesis of CCF is very complex on its genetic aspects. We expect to discover other susceptibility genes, study the association between HOX and other genes, and further study on interaction mechanism among multiple genes is required. We believe that HOX is of great significance in gene therapy and HOX indicates a new direction for the diagnosis and treatment of CCF in the future based on this study.

\section{Acknowledgements}

Not applicable.

\section{Funding}

No funding was received.

\section{Availability data and materials}

The datasets used and/or analyzed during the current study are available from the corresponding author on reasonable request.

\section{Author's contributions}

YW wrote the manuscript, performed RT-PCR and western blot analysis.

\section{Ethics approval and consent to participate}

Signed informed consent forms were obtained from the children's family. The study was approved by the Ethics Committee of The Affiliated Hospital of Southwest Medical University (Luzhou, China).

\section{Consent for publication}

Not applicable.

\section{Competing interests}

Authors declare that they have no competing interests.

\section{References}

1. Ponseti IV: Treatment of congenital club foot. J Bone Joint Surg Am 74: 448-454, 1992.
2. Dobbs MB and Gurnett CA: Update on clubfoot: Etiology and treatment. Clin Orthop Relat Res 467: 1146-1153, 2009.

3. Stewart SF: Club-foot: Its incidence, cause, and treatment; an anatomical-physiological study. J Bone Joint Surg Am 33-A: 577-590, 1951.

4. Kite JH: Principles involved in the treatment of congenital clubfoot. J Bone Joint Surg Am 21: 595-606, 1939.

5. Beeman RW, Stuart JJ, Haas MS and Denell RE: Genetic analysis of the homeotic gene complex (HOM-C) in the beetle Tribolium castaneum. Dev Biol 133: 196-209, 1989.

6. Krumlauf R: Hox genes in vertebrate development. Cell 78: 191-201, 1994.

7. Bienz M: Homeotic genes and positional signalling in the Drosophila viscera. Trends Genet 10: 22-26, 1994.

8. Kite JH: The classic. Principles involved in the treatment of congenital clubfoot by J. Hiram Kite, M.D. reprinted from J. Bone Joint Surg. 21:595-606, 1939. Clin Orthop Relat Res 84: 4-8, 1972.

9. Ponseti IV: Common errors in the treatment of congenital clubfoot. Int Orthop 21: 137-141, 1997.

10. Ponseti IV and Smoley EN: Congenital club foot: The results of treatment. J Bone Joint Surg Am 45: 261-344, 1963.

11. Gerlach DJ, Gurnett CA, Limpaphayom N, Alaee F, Zhang Z, Porter K, Kirchhofer M, Smyth MD and Dobbs MB: Early results of the Ponseti method for the treatment of clubfoot associated with myelomeningocele. J Bone Joint Surg Am 91: 1350-1359, 2009.

12. Pirani S, Zeznik L and Hodges D: Magnetic resonance imaging study of the congenital clubfoot treated with the Ponseti method. J Pediatr Orthop 21: 719-726, 2001.

13. Boehm S, Limpaphayom N, Alaee F, Sinclair MF and Dobbs MB Early results of the Ponseti method for the treatment of clubfoot in distal arthrogryposis. J Bone Joint Surg Am 90: 1501-1507, 2008.

14. Laaveg SJ and Ponseti IV: Long-term results of treatment of congenital club foot. J Bone Joint Surg Am 62: 23-31, 1980.

15. Cummings RJ, Davidson RS, Armstrong PF and Lehman WB: Congenital clubfoot. J Bone Joint Surg Am 84-A: 290-308, 2002

16. Zákány J and Duboule D: Hox genes in digit development and evolution. Cell Tissue Res 296: 19-25, 1999.

17. Arenas-Mena C, Cameron AR and Davidson EH: Spatial expression of Hox cluster genes in the ontogeny of a sea urchin. Development 127: 4631-4643, 2000.

18. Arenas-Mena C, Martinez P, Cameron RA and Davidson EH: Expression of the Hox gene complex in the indirect development of a sea urchin. Proc Natl Acad Sci USA 95: 13062-13067, 1998.

19. Finnerty JR, Pang K, Burton P, Paulson D and Martindale MQ: Origins of bilateral symmetry: Hox and dpp expression in a sea anemone. Science 304: 1335-1337, 2004

20. Castelli-Gair J and Akam M: How the Hox gene Ultrabithorax specifies two different segments: The significance of spatial and temporal regulation within metameres. Development 121: 2973-2982, 1995

This work is licensed under a Creative Commons Attribution-NonCommercial-NoDerivatives 4.0 International (CC BY-NC-ND 4.0) License. 